\title{
New Radiation-Hard Scintillators for FCC Detectors
}

\author{
Y. Onel ${ }^{a, *}$ and B. Bilki ${ }^{a, b}$ \\ a University of Iowa, Iowa City, USA \\ b Beykent University, Istanbul, Turkey \\ E-mail: yasar-onel@uiowa.edu, burak.bilki@cern.ch
}

Future circular and linear colliders including FCC, as well as the Large Hadron Collider in the High-Luminosity era have been imposing unprecedented challenges on the radiation hardness of particle detectors that will be used for specific purposes e.g. forward calorimeters, beam and luminosity monitors. We performed research on the radiation-hard active media for such detectors, particularly calorimeters, by exploring intrinsically radiation-hard materials and their mixtures. The initial samples that we probed were thin plates of Polyethylene Naphthalate (PEN) and Polyethylene Terephthalate (PET) and thin sheets of HEM. The previous studies indicate towards promising performance under high radiation conditions. We report on the necessary process of mixing PEN and PET for optimized scintillation and signal timing properties preserving the high radiation resistance. Recently we developed a new plastic scintillator material, tested it in particle beams and obtained promising results. In recent studies, we investigated the radiation damage to plastic scintillators, various special glasses and scintillating fibers together with their recovery mechanisms. Here we report on the development of radiation-hard scintillators and the irradiation studies together with the investigation of the recovery mechanisms.

The European Physical Society Conference on High Energy Physics (EPS_HEP2021)

26-30 July 2021

Online conference, jointly organized by Universität Hamburg and the research center DESY 


\section{Introduction}

Future and upgrade circular colliders and the projected linear colliders point towards unprecedented radiation conditions on the active media of their detector components, e.g. scintillator tiles and wavelength shifting fibers. In order to address this issue, which is likely to gain further interest in the near future, we have developed radiation-hard scintillator materials and wavelength shifting fibers such as quartz variants with various surface coatings and plastic polymers and elastomers.

Quartz Cerenkov radiators have implementations in beam and luminosity monitors as they are intrinsically radiation-hard. However, they were never considered as active media in calorimetry in the form of quartz plates in a sampling calorimeter since the Cerenkov light yield is insufficient for calorimetry implementations and the light generation is uni-directional. Various light enhancement tools were considered in order to improve the light production inside the quartz plates: pTp (p-Terphenyl), ZnO:Ga (4\% Gallium doped Zinc Oxide), oTp (o-Terphenyl), mTp (mTerphenyl), and pQp (p-Quarterphenyl). Apart from $\mathrm{ZnO}: \mathrm{Ga}$, which needs to be sputtered, all of them can be evaporated in a vacuum chamber to be applied on quartz. Information about relevant radiation-hardness tests performed can be found in [1-3]. Details about the relevant calorimetric measurements can be found in [4].

In terms of intrinsically radiation-hard scintillators, we have investigated thin plates of PEN (Polyethylene Naphthalate) and PET (Polyethylene Terephthalate) as well as thin sheets of HEM. These materials have found previous implementations in beamline instrumentation. We have studied the radiation damage and recovery properties of PEN and PET in great detail with particular emphasis for future calorimetry implementations [5-6].

We developed a new scintillator material with Peroxide- cured polysiloxane bases doped with the primary fluors pTP, pQP, or PPO (2.5-Diphenyloxazole) and/or the secondary fluors 3HF or bis-MSB. We tested the MIP response of the new scintillator at CERN test beam.

Here we report on the performance and radiation-resistance of several scintillators.

\section{Performance Measurement of PEN and PET}

The timing specifications of PEN and PET were measured with $337 \mathrm{~nm}$ pulsed nitrogen laser and also with $120 \mathrm{GeV}$ protons of FTBF [7]. The signal timing in response to the $337 \mathrm{~nm}$ pulsed nitrogen laser is given in Fig. 1, left for PEN and right for PET. The scintillation time constant is measured to be $27.12 \mathrm{~ns}$ for PEN and $6.88 \mathrm{~ns}$ for PET, indicating a significantly faster scintillation for PET. The measurement of signal timing with $120 \mathrm{GeV}$ protons is shown in Fig. 2, left for PEN and right for PET. The time constant for PEN is measured to be $34.91 \mathrm{~ns}$, larger than the laboratory measurement, which is attributed to the differences in the implementation. The time constant for PET is measured to be $6.78 \mathrm{~ns}$, about the same value as the laboratory measurements. On the other hand, PET signals with $120 \mathrm{GeV}$ protons exhibit also a slow time constant which was not observed in the laboratory. For future reference, the $120 \mathrm{GeV}$ proton results serve as the MIP response timing and should have precedence over the laboratory measurements.

PEN and PET MIP response was also measured with $150 \mathrm{GeV}$ muons of CERN test beam [8]. Figure 3 shows the detection efficiency and light yield of PEN (left) and PET (right). PEN 
has an efficiency of approximately $60 \%$ and PET has an efficiency of $10 \%$. Combining the information from the timing measurements, a proper mixture of PEN and PET is expected to yield ideal specifications as a radiation-hard and fast scintillator.
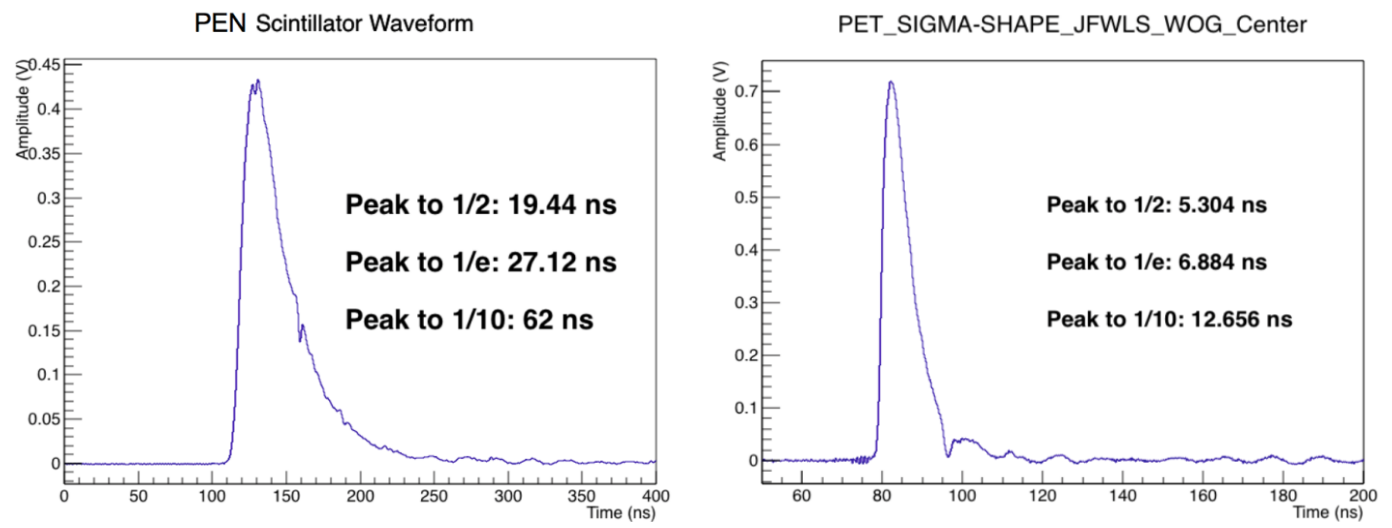

Figure 1. The signal timing of PEN (left) and PET (right) in response to the pulsed nitrogen laser.
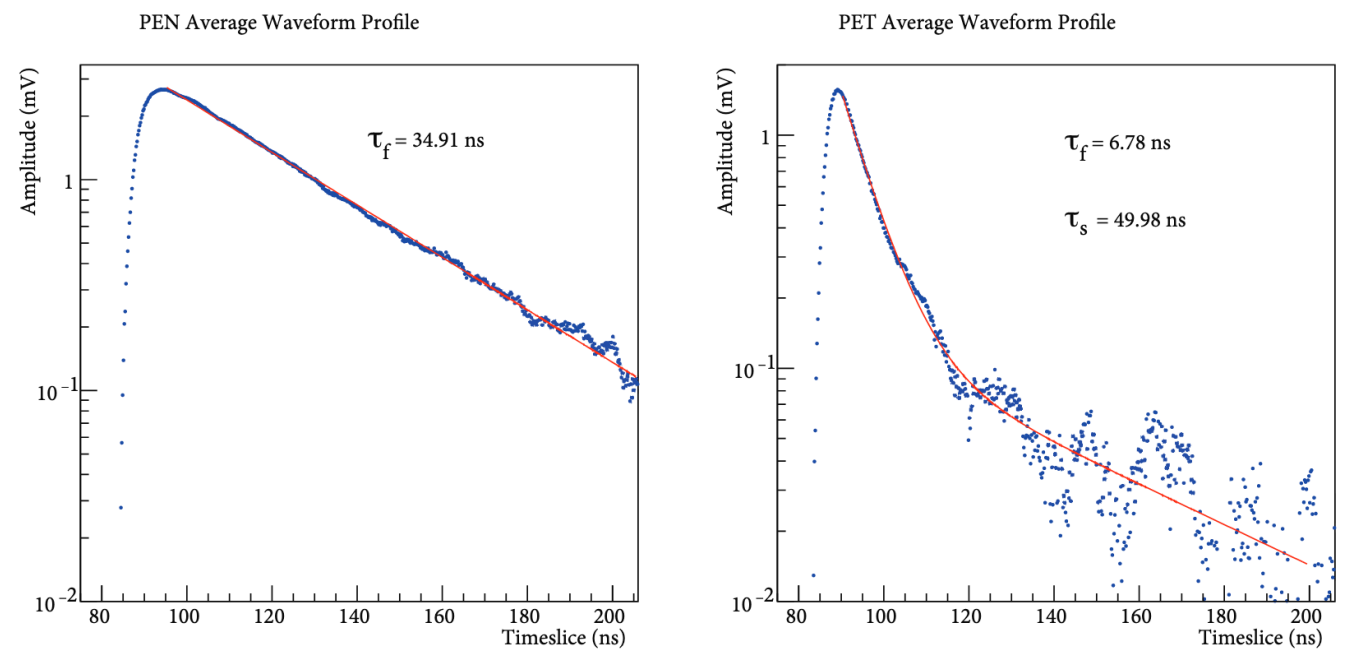

Figure 2. The signal timing of PEN (left) and PET (right) in response to $120 \mathrm{GeV}$ protons of FTBF.
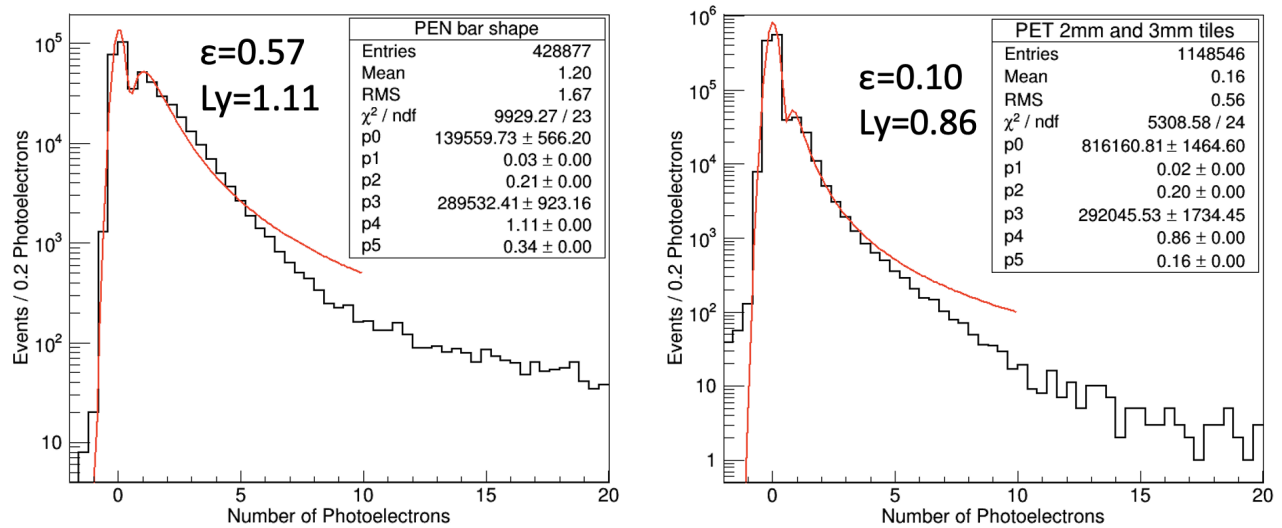

Figure 3. The detection efficiency and light yield of PEN (left) and PET (right) in response to $150 \mathrm{GeV}$ muons of CERN test beam. 


\section{Tests with Plain Quartz Tiles}

A Hamamatsu R7600U-200 PMT was directly coupled to the edge of a combination of clean quartz tiles. This PMT is a new generation PMT and is more sensitive in the UV region compared to the PMT used in the past measurements of MIP response of quartz tiles. Figure 4 (left) shows the response of a single tile with reflective wrapping (black), a stack of five tiles with no wrapping (red) and a stack of five tiles with individual reflective wrappings to $120 \mathrm{GeV}$ protons of FTBF. The change in response is obvious in Fig. 4 (left). Then, stacks of up to 18 tiles, all with individual wrapping, were exposed to the proton beam. A linear relation was observed between the most probable value of the MIP response of the stack and the number of tiles in the stack (Fig. 4 right). The conclusion of this study is that the quartz plate calorimetry should be revisited with the new generation photodetectors which are more sensitive in the UV region.
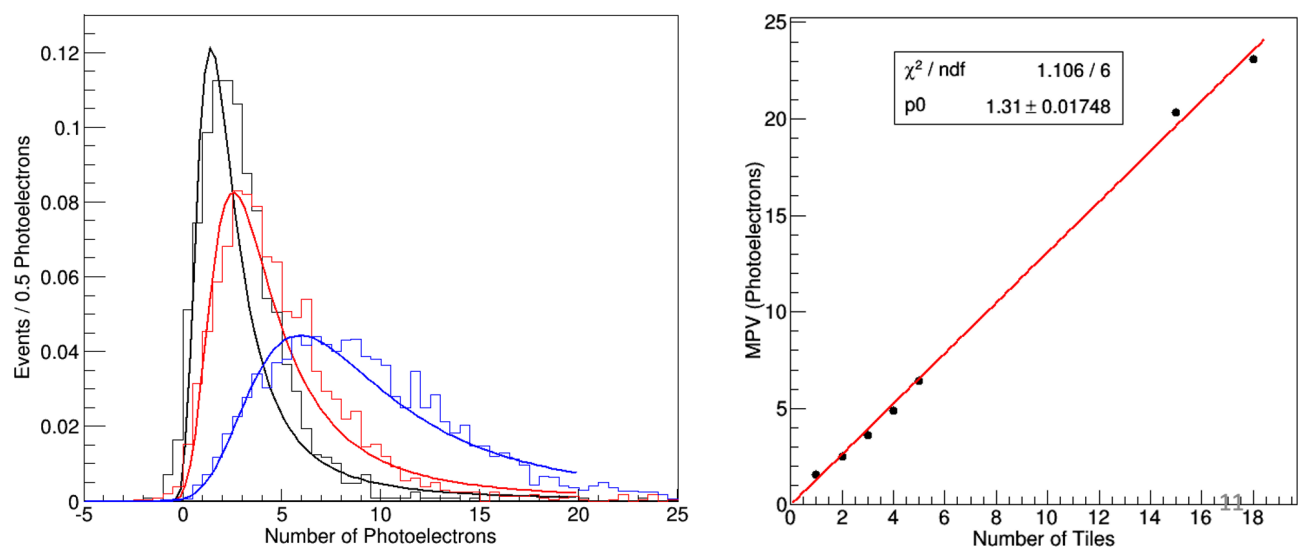

Figure 4. The response of a single tile with reflective wrapping (black), a stack of five tiles with no wrapping (red) and a stack of five tiles with individual reflective wrappings to $120 \mathrm{GeV}$ protons of FTBF (left), and the most probable response as a function of the number of tiles in the stack (right).

\section{Tests with the New Scintillator}

Recently, we developed many samples of the polysiloxane based scintillators and tested the scintillators for their response in $150 \mathrm{GeV}$ muons of CERN test beam. Two $3 \mathrm{~cm} \times 3 \mathrm{~cm}$ tiles (one of which was polished) with photodetectors (Hamamatsu S12572-10 [9]) directly coupled to the dimples were tested at CERN $\mathrm{H} 2$ beam line with $150 \mathrm{GeV}$ muon beam to measure the response of the tiles to Minimum Ionizing Particles (MIPs). The SiPMs were downstream and the tiles were centered with respect to the beam. The lateral size of the beam was much larger than the size of the SiPM, and the muons passing through the tile and $1 \mathrm{~mm}$ away from the SiPM location were selected using wire chamber profiles. Figure 5 shows the charge spectrum of the polished (left) and unpolished (right) scintillators in response to traversing MIPs. The distributions were fit to Gaussian + Landau. The mean for the Gaussian and the most probable value for the Landau functions were constrained to be identical, denoted as $\mu$ in Fig. 5. The mean response of the polished tile to MIPs corresponds to approximately 18 photons, and that of the unpolished one corresponds to approximately 14 photons. With these results, the recent production process 
modifications are validated. The production procedure can be extended to various currently unprobed specifications.
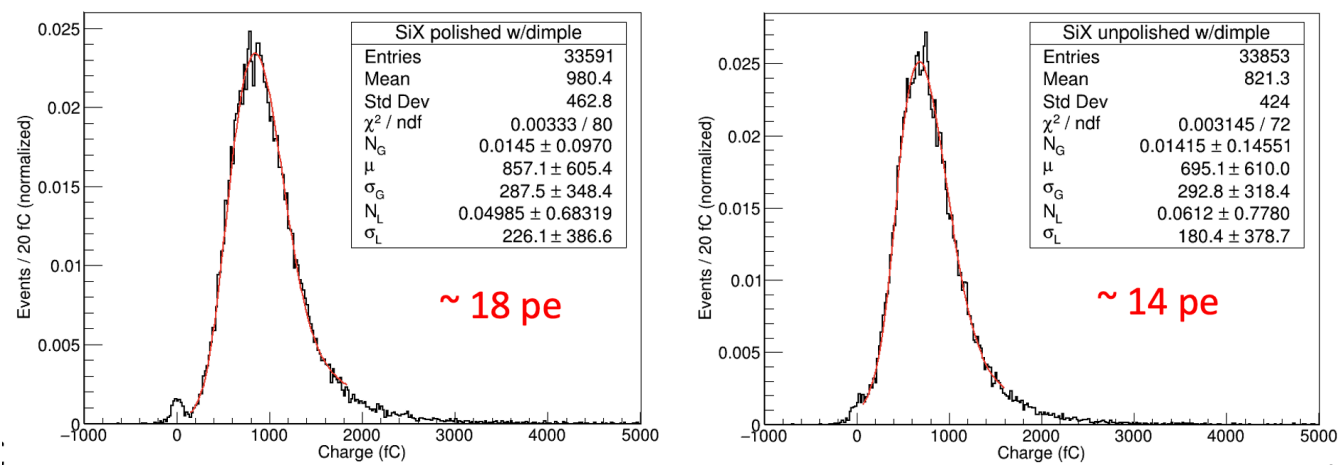

Figure 5. The charge spectrum of the polished (left) and unpolished (right) scintillators in response to traversing MIPs.

\section{The Radiation Damage and Recovery of PEN and PET}

PEN and PET samples were also irradiated to $1.4 \mathrm{Mrad}$ and $14 \mathrm{Mrad}$ with gammas at the Iowa RadCore Facility [10]. Figure 6 shows the recovery curves of $1.4 \mathrm{Mrad}$ (left) and $14 \mathrm{Mrad}$ (right) irradiated PEN samples. The initial damage in the $1.4 \mathrm{Mrad}$ irradiated sample is slightly less that $30 \%$ whereas that of 14 Mrad irradiated sample is above $50 \%$. Despite an order of magnitude increase in the total absorbed dose, the initial damage does not scale comparably.
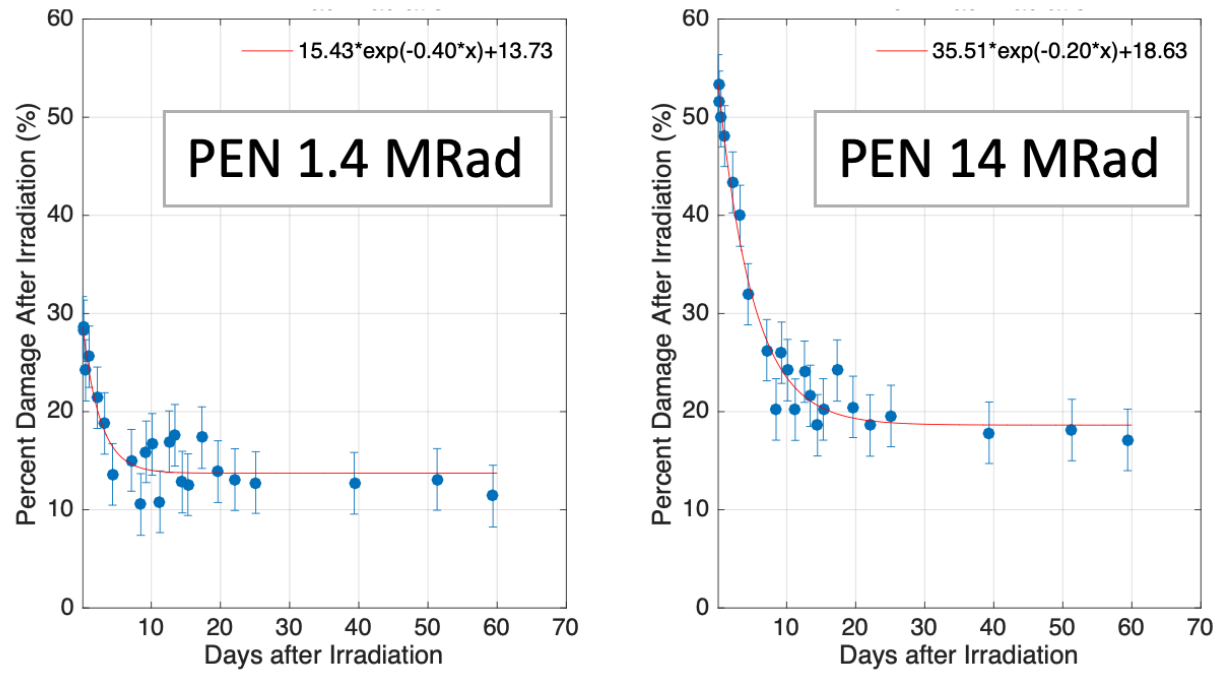

Figure 6. The recovery curves of $1.4 \mathrm{Mrad}$ (left) and $14 \mathrm{Mrad}$ (right) irradiated PEN samples from radiation damage.

Figure 7 shows the same set of curves for PET. The initial damage is observed to be considerably higher that that of PEN, and as a result, the recovery also takes longer. The permanent damage on the other hand is comparable for PEN and PET. 

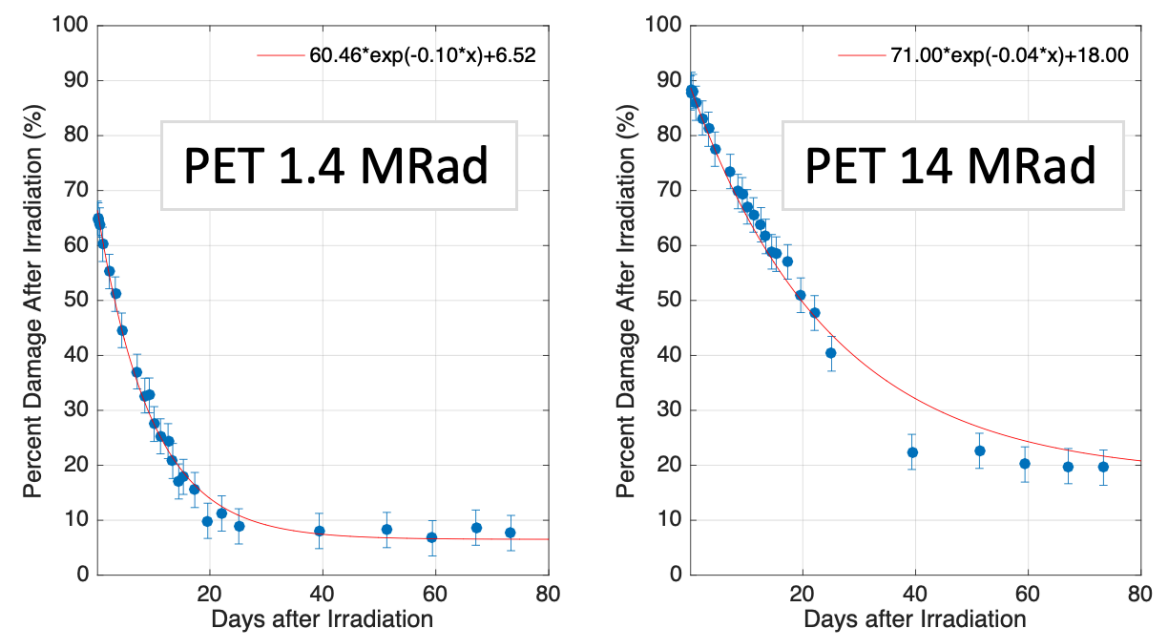

Figure 7. The recovery curves of $1.4 \mathrm{Mrad}$ (left) and $14 \mathrm{Mrad}$ (right) irradiated PET samples from radiation damage.

\section{Conclusions}

We have probed several techniques to develop radiation-hard scintillators and the progress on all grounds is promising. For the timescale of the FCC detectors, the developments are expected to be a much more mature. A few immediate observations include the necessity of mixing of PEN and PET to obtain more desirable scintillation, timing and radiation-hardness parameters; and the necessity of further investigation of the new scintillator development options to yield optimization for mass production.

\section{References}

[1] I. Dumanoglu, et al., Nucl. Instrum. Meth. A490 444, 2002.

[2] K. Cankocak, et al., Nucl. Instrum. Meth. A585 20, 2008.

[3] U. Akgun, et.al., AIP Conf.Proc. 867 282, 2006.

[4] U. Akgun, et.al., IEEE Trans. on Nucl. Sci. 57-2 754, 2010.

[5] J. Wetzel, et.al. JINST 11 P08023, 2016.

[6] J. Wetzel, et.al., Nucl. Instrum. Meth. B395, 13-16, 2017.

[7] https://ftbf.fnal.gov/

[8] http://sba.web.cern.ch/sba/

[9] https://www.hamamatsu.com

[10] https://frrbp.medicine.uiowa.edu/research-core 